\title{
COGROWTH AND ESSENTIALITY IN GROUPS AND ALGEBRAS
}

\author{
Amnon Rosenmann* \\ Institute for Experimental Mathematics \\ University GH Essen \\ 45326 Essen, Germany \\ amnon@exp-math.uni-essen.de
}

\begin{abstract}
The cogrowth of a subgroup is defined as the growth of a set of coset representatives which are of minimal length. A subgroup is essential if it intersects non-trivially every non-trivial subgroup. The main result of this paper is that every function $f: \mathbf{N} \cup\{0\} \longrightarrow \mathbf{N}$ which is strictly increasing, but at most exponential, is equivalent to a cogrowth function of an essential subgroup of infinite index of the free group of rank two. This class of functions properly contains the class of growth functions of groups.

The notions of growth and cogrowth of right ideals in algebras are introduced. We show that when the algebra is without zero divisors then every right ideal, whose cogrowth is less than that of the algebra, is essential.
\end{abstract}

\section{Growth, Cogrowth and Essentiality in Groups}

\subsection{Growth and Cogrowth of Subgroups}

A growth function $\Gamma_{S}(n)$ on a set $S$ with a length function $l$ on it is defined by

$$
\Gamma_{S}(n):=\operatorname{card}\{s \in S \mid l(s) \leq n\},
$$

assuming that $\Gamma_{S}(n)$ is finite for each $n$. A preorder is given on the growth functions by

$$
\Gamma_{1}(n) \preceq \Gamma_{2}(n) \Longleftrightarrow \exists C\left[\Gamma_{1}(n) \leq \Gamma_{2}(C n)\right] .
$$

The notion of growth when applied to finitely generated groups (see $[6]$ for an overview) has been investigated mainly after Milnor's paper ([12]). A geometric interpretation can be given, for example, when computing the growth function

*Supported by the Minerva Fellowship 
of the fundamental group of a Riemannian manifold. In order to avoid the dependence of the length function upon the generating set of the group, an equivalence relation is used

$$
\Gamma_{1}(n) \sim \Gamma_{2}(n) \Longleftrightarrow \exists C\left[\Gamma_{1}(n) \leq \Gamma_{2}(C n) \& \Gamma_{2}(n) \leq \Gamma_{1}(C n)\right] .
$$

We will also use the following notation

$$
\Gamma_{1}(n) \prec \Gamma_{2}(n) \Longleftrightarrow \Gamma_{1}(n) \preceq \Gamma_{2}(n) \& \Gamma_{1}(n) \nsim \Gamma_{2}(n) .
$$

The growth function of the group $G$ will be denoted by $\Gamma_{G}(n)$, when referring to its equivalence class and also when some fixed generating set is assumed (but omitted in the notation).

When $H$ is a subgroup of $G$ we may speak of the cogrowth of $H$ in $G$, denoted $\Gamma_{G / H}(n)$. This is defined to be the growth of a (complete) set of coset representatives for $H$ in $G$ which is "minimal" in the sense that every representative is of minimal length in its coset relative to the given group-generating set of $G$. Clearly, any other set of coset representatives will grow at most as fast as a minimal set. We also notice that cogrowth functions of $H$ relative to different generating sets of $G$ are equivalent. (Remark: it does not matter if we take right or left cosets because the inverses of the right representatives can be used as left representatives). Constructing the set of right coset representatives by induction on length, we see that it can always be chosen so that its elements are initially closed, that is arranged in a form of a tree. Such a set is called a Schreier transversal (see [11]). Presenting an order on the generating set of $G$ induces a "ShortLex" total order on $G$ (comparing elements first by length and then by the lexicographic order). Relative to this order each subgroup has a unique minimal transversal, which is also a Schreier transversal.

The growth of a subgroup $H<G$ with respect to the generators of $G$ was studied by Grigorchuk (see 四). We denote this growth function by $\Gamma_{H}^{(G)}(n)$ (to distinguish it from the growth function of $H$ when considered a group). A connection between the different growth functions can be given by (assuming a fixed generating set)

$$
\sum_{i=0}^{n} \gamma_{G / H}(i) \Gamma_{H}^{(G)}(n-i) \leq \Gamma_{G}(n) \leq \sum_{i=0}^{n} \gamma_{G / H}(i) \Gamma_{H}^{(G)}(n+i),
$$

where $\gamma_{G / H}(i):=\Gamma_{G / H}(i)-\Gamma_{G / H}(i-1)$, that is the coset representatives of length exactly $i$. The left inequality comes from the fact that different cosets are disjoint subsets. The right inequality is by the definition of the cogrowth function through the coset representatives of minimal length.

When $H$ is a normal subgroup of $G$ then the cogrowth of $H$ describes the growth of the group $G / H$. This imposes restrictions on the cogrowth of $H$ :

$$
\Gamma_{G / H}\left(n_{1}+n_{2}\right) \leq \Gamma_{G / H}\left(n_{1}-1\right)+\gamma_{G / H}\left(n_{1}\right) \Gamma_{G / H}\left(n_{2}\right) .
$$


Since each right coset is also a left one when $H$ is normal, the minimal Schreier transversal tree $T$, relative to a ShortLex order, is suffix as well as prefix-closed. In other words, each subtree $T^{\prime}$ of $T$ is "covered" by $T$ when putting the root of $T$ over the root of $T^{\prime}$. (This is also the reason why a finitely generated subgroup of a free group which contains a non-trivial normal subgroup is of finite index.) Thus if $g=g_{1} g_{2} \in T$ when written in reduced form then the subtree $T(g)$ with root in $g$ is "contained" (in the above sense) in the subtree $T\left(g_{2}\right)$. Therefore, there exists a descending chain of subtrees of $T$, which is of length $l(g)$ and terminates with $T(g)$. This tendency of $T$ to "close" itself raised the question if there are groups of non-exponential but also non-polynomial growth. Milnor and Wolf showed that a f.g. solvable group has polynomial growth if it is virtually-nilpotent and otherwise has exponential growth ([13], [16]). Gromov ([4]) showed that virtually-nilpotent groups are the only ones with polynomial growth. Then Grigorchuk succeeded to obtain remarkable examples of groups of "intermediate growth" (between $n^{d}$ and $e^{n}$ ), and to show that the set of growth degrees of finitely generated groups is of the continuum cardinality (see [5], [6] and also [3]).

If $H_{1}, H_{2}$ are subgroups of $G$ then $\Gamma_{G / H_{1} \cap H_{2}}(n) \geq \max \left\{\Gamma_{G / H_{1}}(n), \Gamma_{G / H_{2}}(n)\right\}$ for every $n$ (as usual, we assume here that the generating set of $G$ is fixed). We also know that the intersection of two subgroups of finite index can be at most of the product of the indices. But in fact $\Gamma_{G / H_{1} \cap H_{2}}$ behaves in this manner all along the way.

Proposition 1.1 If $H_{1}, H_{2}<G$ then $\Gamma_{G / H_{1} \cap H_{2}}(n) \leq \Gamma_{G / H_{1}}(n) \Gamma_{G / H_{2}}(n)$ for every $n$.

Proof. Let $T_{1}, T_{2}$ and $T$ be minimal right Schreier transversals for $H_{1}, H_{2}$ and $H_{1} \cap H_{2}$ respectively. Each element $g \in T$ can be represented by a pair $\left(g_{1}, g_{2}\right)$, $g_{i} \in T_{i}$, where $g_{i}$ is the representative of the coset $H_{i} g$ of $H_{i}$. By the minimality of the lengths of the coset representatives, $l\left(g_{i}\right) \leq l(g)$ for each $i$. The result then follows since the pairs are distinct.

The proposition gives a sufficient condition for the intersection of two subgroups to be non-trivial. $\Gamma_{G / H_{1}}(n) \Gamma_{G / H_{2}}(n) \prec \Gamma_{G}(n) \Longrightarrow \Gamma_{G}(n) \npreceq \Gamma_{G / H_{1}}(n) \Gamma_{G / H_{2}}(n) \Longrightarrow$ $\exists n\left[\Gamma_{G / H_{1}}(n) \Gamma_{G / H_{2}}(n)<\Gamma_{G}(n)\right] \Longrightarrow H_{1} \cap H_{2}$ is non-trivial. For example, if $\Gamma_{G}(n) \sim d^{n^{e}}$, where $d>1,0<e \leq 1$, and $\Gamma_{G / H_{1}}(n), \Gamma_{G / H_{2}}(n) \prec \Gamma_{G}(n)$ then $H_{1} \cap H_{2}$ is non-trivial.

If $H_{2}<H_{1}<G$ then an element $g \in G$ of minimal length in $H_{1} g$ is also of minimal length in the coset $H_{2} g$, thus a minimal Schreier transversal for $H_{1}$ can be chosen to be a subset of a minimal transversal for $H_{2}$.

Let $1 \longrightarrow H \stackrel{i}{\longrightarrow} F \stackrel{\pi}{\longrightarrow} G \longrightarrow 1$, where $F$ is free and finitely generated on $X$. Then there is a 1-1 correspondence between a minimal Schreier transversal $T$ for $i(H)$ in $F$ and the set of elements of the group $G$ with generating set $\{\pi(x) \mid x \in X\}$, given by $g=x_{i_{1}} \cdots x_{i_{n}} \longleftrightarrow \pi(g)=\pi\left(x_{i_{1}}\right) \cdots \pi\left(x_{i_{n}}\right)$, where $g$ and $\pi(g)$ here are 
already in reduced form (note that we do not require the extension to split). Then a (minimal) Schreier transversal for a subgroup $G^{\prime}$ of $G$ can be represented (with the above correspondence) by a (minimal) Schreier transversal for $\pi^{-1}\left(G^{\prime}\right)$ in $F$ which can be taken to be a subset of $T$. This means that the cogrowth functions of subgroups of finitely generated groups are all cogrowth functions of subgroups of finitely generated free groups (and as will be seen in Theorem 1.2, it suffices to consider the free group on 2 generators for the equivalence classes of the cogrowth functions).

\subsection{Essential Subgroups and Their Cogrowth Functions}

We come now to essential subgroups. We call a subgroup $H<G$ essential if it intersects non-trivially every non-trivial subgroup of $G$. Clearly the family $\mathcal{E}$ of essential subgroups of a given group is a filter: if $H_{1} \in \mathcal{E}$ and $H_{1}<H_{2}$ then $H_{2} \in \mathcal{E}$, and if $H_{1}, H_{2} \in \mathcal{E}$ then $H_{1} \cap H_{2} \in \mathcal{E}$. Also a conjugate of an essential subgroup is essential. For example, if $G$ is torsion-free then clearly every subgroup of finite index is essential. In finitely generated free groups, a subgroup is of finite index if and only if it is finitely generated and essential.

In contrast to the situation in algebras (as will be shown in the next section), one cannot tell whether a subgroup of infinite index of a free group is essential or not just by knowing the cogrowth of the subgroup. This is due to the "onedimensionality" of a subgroup generated by a single element. For example, let $G$ be the free group on the two generators $x, y$ and let $H$ be the normal closure of the subgroup of $G$ generated by $x$. Then a minimal Schreier transversal for $H$ consists of all powers of $y$, hence $\Gamma_{G / H}(n) \sim n$. But $H$ is not essential since $H \cap\langle y\rangle=1$. Thus, when $H$ is of infinite index it can be of minimal cogrowth and still lack essentiality. On the other hand, $H$ can be essential although it has exponential cogrowth. If $H$ is a normal subgroup of a torsion-free group $G$ then by definition $H$ is essential if and only if $G / H$ is periodic (torsion). The well known examples of essential subgroups $H \triangleleft G$ of exponential cogrowth are when $G$ is free of rank $m \geq 2$ and $G / H$ is the Burnside group $B(m, n)$ with $n \geq 665$ and odd, as shown by Adyan ([1]). Essential normal subgroups of intermediate growth were constructed by Grigorchuk.

Let $\mathcal{C G}$ be the class of functions $\alpha(n)$ of the following type. $\alpha: \mathbf{N} \cup\{0\} \longrightarrow \mathbf{N}$ is the sequence of partial sums $\sum_{i=0}^{n} f_{i}$ of the series $\sum_{i=0}^{\infty} f_{i}$, such that (i) the $f_{i^{-}}$ $\mathrm{s}$ are zero on $(r, \infty)$, where $0 \leq r$ and can be $\infty$, and positive integers otherwise, with $f_{0}=1$; (ii) there exists $0<d$ such that $f_{i+1} \leq d f_{i}$ for every $i$. Clearly $\mathcal{C G}$ includes the cogrowth functions of subgroups, but as seen from (id), the set of equivalence classes of the growth functions of groups is properly contained in the set of equivalence classes of the members of $\mathcal{C G}$.

Theorem 1.2 Let $G$ be the free group on $X=\left\{x_{1}, x_{2}\right\}$. Then for every $\alpha(n) \in$ $\mathcal{C G}$ there exists an essential subgroup $H$ of $G$ such that $\Gamma_{G / H}(n) \sim \alpha(n)$.

Proof. If $\alpha(n)$ is eventually-constant then any subgroup of finite index can be 
taken. So let us assume that $\alpha(n)$ is not bounded. We will construct in an inductive way a right Schreier transversal $T$ for $H$. $T$ will contain two types of sections constructed alternately: those responsible for the desired growth (gsections), and those to ensure the essentiality (e-sections). The growth function of $T, \Gamma_{T}(n)$, will be $\preceq \Gamma_{G / H}(n)$, which is the growth function of a minimal Schreier transversal tree, but by an appropriate definition of the coset function, and by letting the g-sections be of sufficient depth (length) compared to the e-sections, $T$ can be constructed such that $\Gamma_{T}(n) \sim \Gamma_{G / H}(n)$ (if each g-section will be of depth equal to that of the next e-section, the growth of $T$ will be at least as half the growth of $\left.\Gamma_{G / H}\right)$.

We start with a g-section. Here we prevent the occurrence of the same generator (or the same inverse of a generator) in adjacent edges. Hence, each non-root vertex will have either 1 or 2 out-going edges. As we will see later, an essential section can be constructed to be of growth $2 n$, so no problem will be to achieve growth equivalent to the minimal possible growth of $\alpha(n)$. As for the maximal growth, $\alpha(n)$ can grow at most as $d^{n}$, for some positive $d$. So if $c$ is such that $2^{c} \geq d$ then $2^{c n} \geq d^{n}$, and we can construct $T$ such that $\alpha(n) \leq \Gamma_{T}(c n)$. Then this inequality can surely be reached when $\alpha(n)$ grows slower than $d^{n}$, and thus $\Gamma_{T}(n)$ can be bounded by

$$
\alpha\left(c^{-1} n\right) \leq \Gamma_{T}(n) \leq \alpha(2 n) .
$$

(In general, $\Gamma_{T}(n)$ can be constructed so that it grows at the fastest possible rate, but not exceeding $\alpha(n)$, resulting in some averaging of $\alpha(n)$, making it "smoother" in places of great jumps.)

Suppose we constructed the tree $T$ up to depth $p_{1}$, being the first g-section. We label also each edge of $T$ by some $x \in X \cup X^{-1}$, such that the labels on the vertices are the elements one obtains by reading off the edge labels in a path that starts at the root and terminates at the given vertex. We then partially define the coset function $\pi$. If an edge labelled by $x \in X \cup X^{-1}$ goes from the vertex $g$ to the vertex $h$ then $\pi(g x)=h$ and $\pi\left(h x^{-1}\right)=g$, that is $\pi(g)=g$ for every $g \in T$. Otherwise, we define $\pi$ on the set

$$
\left\{g x \mid g \in T, l(g)<p_{1}-1 \text { and } x \in X \cup X^{-1}\right\}
$$

in the following way. We go as far as possible on a path in the opposite direction. That is, we start at $g$, and go from a vertex $h^{\prime}$ to a vertex $h^{\prime \prime}$ whenever $\pi\left(h^{\prime} x^{-1}\right)$ is defined and $h^{\prime \prime}=\pi\left(h^{\prime} x^{-1}\right)$. If the vertex $h$ is the endpoint of this path (in general, $h$ can be $g$ itself) then we define $\pi(g x)=h$. We notice that by the restriction of not labeling two adjacent edges with the same letter, such a path as described above will be of length 1 (except near the root where it can be of length 2). The same process of defining $\pi$ will take place at the following stages, but then, due to the e-sections, paths as above could be of greater length. The definition of $\pi$ on other elements of $G$ is then according to the inductive rule $\pi(g x)=\pi(\pi(g) x)$. 
Next we construct an e-section. Assume an ordered list of the elements of $G$ is given. We take the first element $g$ of this list, and "travel" along $T$ as long as possible with powers of $g$, starting from the root 1 and using the function $\pi$. If we happen to get back to the root after some $k$-th power of $g$ then we are done: $g^{k} \in H$, and we can take the next element in the list. Otherwise, we will extend $T$ and $\pi$ so that $\langle g\rangle$ will intersect $H$ non-trivially. (One may look at $T$ as representing an automaton, which has to be extended so that $g$ will be accepted by it. Here the states of the automaton are the vertices, with 1 the accepting state, the input alphabet is $X \cup X^{-1}$ and the function is $\pi$.) The idea is to form two paths starting from the root, one of a positive power of $g$ and the other of a negative power, and to "tie" these paths using the function $\pi$. Assume that when written in reduced form we have $g=h_{1} h_{2} h_{1}^{-1}$, where $h_{2}$ is of minimal length. We start a travel from the root of $T$, this time with $h_{1}$ followed by powers of $h_{2}$. It may still happen that we will return to $\pi\left(h_{1}\right)$ after some powers of $h_{2}$, and in this case too we are done. (We note that it is impossible to return to the same vertex after some powers of $h_{2}$ before first visiting $\pi\left(h_{1}\right)$. This is because by the very definition of $\pi$, if $\pi\left(h_{1} h_{2}^{r}\right)=\pi\left(h_{1} h_{2}^{s}\right)$ then $\pi\left(h_{1} h_{2}^{s-r}\right)=\pi\left(h_{1}\right)$.) Otherwise, we reach a vertex $h \in T$ on which $\pi(h x)$ is not yet defined, where $x$ is the next "input" letter of $h_{1} h_{2}^{k}$ for some $k \geq 0$. We then increase $T$ by adding a path, starting with the vertex $h x$, according to the rest of $h_{1} h_{2}^{k}$. The same process of increasing the tree is then done with a path that goes from the root with $h_{1}$ followed by powers of $h_{2}^{-1}$. Since no prefix of $h_{2}^{-1}$ equals a suffix of $h_{2}$, the two added paths must become separated one from another, and after that happens we need not increase the tree anymore. The endpoints of the two added paths are vertices $u_{1}$ and $u_{2}$ such that $u_{1}=\pi\left(h_{1} h_{2}^{r}\right)$ and $u_{2}=\pi\left(h_{1} h_{2}^{-s}\right)$, for some $r, s \geq 0$. Assume now that $h_{2}=x w$, where $x \in X \cup X^{-1}, w \in G$ and $h_{2}$ is written in reduced form. Then we further extend the tree by adding a $w^{-1}$-segment at the vertex $u_{2}$ and define $\pi\left(u_{1} x\right)=u_{2} w^{-1}$, and necessarily $\pi\left(u_{2} w^{-1} x^{-1}\right)=u_{1}$. Later, this construction will result (after a sufficient extension of $\pi$ ) in

$$
\pi\left(g^{r+s+1}\right)=1,
$$

i.e. $\langle g\rangle$ will intersect $H$ non-trivially. Let us call $T_{1}$ the current tree we have.

Next we construct a g-section, extending $T_{1}$ and the coset function $\pi$, according to the growth function $\alpha(n)$, the same as before. If needed, we first widen the tree in the part of the e-section to reach the desired growth, but we do not change $\pi$ where it is already defined. Again, we do not define $\pi$ on the boundary of the current tree, to ensure further increasing of the tree. Following this stage comes an e-section with the next element in the list. The result is a tree $T_{2}$. We continue in this way indefinitely and define $T=\bigcup_{i} T_{i}$. Clearly

$$
\pi\left(\pi(g x) x^{-1}\right)=g
$$

for every $g \in T, x \in X \cup X^{-1}$. This makes $T$ a right Schreier transversal for a unique subgroup $H$ of $G$, for which $\pi$ is the function giving the coset 
representatives (see [8], [9]). $H$ is freely generated by the non-trivial elements of the form $g x(\pi(g x))^{-1}$, where $g \in T, x \in X$ (Nielsen-Schreier theorem).

As said before, $\Gamma_{T}(n)$ can be made equivalent to $\alpha(n)$. On the other hand, if $g$ is a vertex in a g-section (and assume it is not on the boundary of the section) then $l(\pi(g x)) \leq l(g)+1$, for any $x \in X \cup X^{-1}$. If $g$ is in an e-section then $l(\pi(g x)) \leq l(g)+r$, where $r$ is the length of the e-section. Therefore, if each g-section has at least the depth of the next e-section, we get for any $g \in G$

$$
l(\pi(g)) \leq 2 l(g)
$$

Thus, the length of each element in $T$ is at most twice the length of the minimal element in its coset, and so $\Gamma_{G / H}(n) \leq \Gamma_{T}(2 n)$ and they are equivalent (because $\left.\Gamma_{T}(n) \leq \Gamma_{G / H}(n)\right)$. Combining it with (7) gives

$$
\Gamma_{G / H}(n) \sim \Gamma_{T}(n) \sim \alpha(n) .
$$

Finally, the e-sections make sure that for every element of $G$ some positive power of it lies in $H$, that is $H$ is essential.

We remark that with a little more effort (by adding segments corresponding to the different group elements), the subgroups constructed in the theorem above can have the additional property of not containing any subgroup which is normal in $G$.

As we have seen, even when the group is torsion-free a normal subgroup of infinite index need not be essential although it can be of minimal cogrowth. However, the following simple observation expresses the "largeness" of normal subgroups in special cases. If $G$ is a torsion-free group which does not contain a non-cyclic abelian subgroup then every two non-trivial normal subgroups of $G$ have non-trivial intersection. To see it, let $1 \neq x \in H_{1}, 1 \neq y \in H_{2}$, where $H_{1}$ and $H_{2}$ are normal subgroups of $G$. Then $x y x^{-1} y^{-1}=x\left(y x^{-1} y^{-1}\right) \in H_{1}$ and also $x y x^{-1} y^{-1}=\left(x y x^{-1}\right) y^{-1} \in H_{2}$ and the result follows.

\section{Growth, Cogrowth and Essentiality in Alge- bras}

Let $R$ be an associative algebra with a unit generated on a finite set $X$ over a field $K$. Having the length function on the free semigroup $X^{*}$ generated by $X$ and the grading of $R$ by the subspaces $R^{(n)}=\sum_{i=0}^{n} K X^{i}$, the growth function on $R$ is defined by

$$
\Gamma_{R}(n):=\operatorname{dim} R^{(n)}
$$

(see [15], and also [2] for a generalization). The length of an element $r \in R$ is the smallest $n$ such that $r \in R^{(n)}$. As in groups, the equivalence class of a growth function does not depend upon the set of generators. 
If $I$ is a right ideal of $R$ then its growth function is $\Gamma_{I}(n):=\operatorname{dim}\left(I \cap R^{(n)}\right)$, and its cogrowth $\Gamma_{R / I}(n):=\Gamma_{R}(n)-\Gamma_{I}(n)$. The definition can then be extended to subspaces of $R$ by $\Gamma_{V}(n):=\operatorname{dim}\left(V \cap R^{(n)}\right)$, and if $V \supseteq U$ then $\Gamma_{V / U}(n):=$ $\Gamma_{V}(n)-\Gamma_{U}(n)$.

Let $V$ be a complementary subspace to $I$, that is $R=I+V$ and $I \cap V=0$. Then a basis $T$ for $V$ can consist of a set of (monic) monomials, which moreover is initially closed, that is forms a tree. Introducing a ShortLex order on $X^{*}$ and extending it in the usual manner to a partial order on $R$, such a basis can be formed from all monomials which are minimal in their cosets (see [10]), resulting in a unique minimal Schreier transversal $T$ (similar to the group case). Then we get

$$
\begin{aligned}
\Gamma_{I}(n) & =\operatorname{card}\{g \mid g \text { is a leading monomial of some } r \in I, l(r) \leq n\}(, 14) \\
\Gamma_{R / I}(n) & =\operatorname{card}\{g \in T \mid l(g) \leq n\} .
\end{aligned}
$$

In fact, when $R$ is a group algebra $K G$ and $H$ a subgroup of $G$ then the Schreier transversals for the right ideal $I$ of $R$ generated by the elements $h-1$, where $h \in H$, coincide with the Schreier transversals for $H$ in $G$, and thus $\Gamma_{G / H}(n)=$ $\Gamma_{R / I}(n)$ (keeping the generating set for $G$ fixed).

The situation concerning intersection of right ideals is simpler than intersection of subgroups because in each $R^{(n)}$ we have intersection of finite dimensional subspaces.

If $I$ is a right ideal and $r \in R$ then $(I: r):=\{s \in R \mid r s \in I\}$.

Proposition 2.1 If $\Gamma_{I}(n) \npreceq \Gamma_{R / I}(n)$ then for every $0 \neq r \in R,(I: r) \neq 0$.

Proof. We may assume that $l(r) \geq 1$. Since $\Gamma_{I}(n) \npreceq \Gamma_{R / I}(n)$ then

$$
\forall C \exists n\left[\Gamma_{R}(n) \geq \Gamma_{I}(n)>\Gamma_{R / I}(C n) \geq \Gamma_{r R+I / I}(C n)\right] .
$$

Hence $\Gamma_{r R+I / I}(n) \prec \Gamma_{R}(n)$. Taking $C=2 l(r)$ we get $C n \geq n+l(r)$ and by (16)

$$
\exists n\left[\Gamma_{R}(n)>\Gamma_{r R+I / I}(n+l(r))\right] .
$$

We look now at $\left\{r g \mid g \in G, l(g) \leq n_{0}\right\}$, where $n_{0}$ is such that the inequality in (17) holds. We have here $\Gamma_{R}\left(n_{0}\right)$ elements of length $\leq n_{0}+l(r)$. By (17) these elements are linearly dependent modulo $I$, hence there exists some $0 \neq s=\sum_{l(g) \leq n_{0}} a_{g} g, a_{g} \in K, g \in G$, such that $r s \in I$, i.e. $(I: r) \neq 0$.

We now come to essentiality of right ideals. Essential right ideals are more common than essential subgroups. ¿From a geometrical point of view, the difference is that a right ideal generated by a single element grows in a cone-like manner, whereas cyclic subgroups are "1-dimensional".

Corollary 2.2 Let $R$ be without zero divisors and let $I$ be a right ideal of $R$. If $\Gamma_{R / I}(n) \prec \Gamma_{R}(n)$ then $I$ is essential. 
Proof. Since $I$ is not empty it contains a right regular element. Therefore its growth function is equivalent to the growth function of $R$. Thus $\Gamma_{R / I}(n) \prec$ $\Gamma_{I}(n)$, and by Proposition $2.1 I$ is essential.

Remark: The converse of the above does not hold.

When $R$ is the group algebra $K G$, where $G$ is free of rank 2 and $K$ is a field, then for every $\alpha(n) \in \mathcal{C G}$ (as defined in the previous section) there exists an essential right ideal $I$ of $R$ with $\Gamma_{R / I}(n) \sim \alpha(n)$. For the functions which are $\prec 2^{n}$ we can take the right ideal generated by the "right augmentation ideal" of the subgroups constructed in Theorem 1.2. For exponential growth we can take, for example, the fractal ideals defined in 14].

\section{References}

[1] Adyan, S.I. (1975). The Burnside problem and identities in groups. Nauka, Moscow, 1975 (Russian) [(1979) Ergebnisse der Mathematik und ihrer Grenzgebiete, 95. Springer-Verlag].

[2] Aljadeff, E., Rosset, S. (1988). Growth and uniqueness of rank. Israel J. Math., Vol. 64, No. 2, 251-256.

[3] Fabrykowski, J., Gupta, N. (1985). On groups with sub-exponential growth functions. J. Indian Math. Soc., Vol 49, 249-256.

[4] Grigorchuk, R.I. (1978). Symmetrical random walks on discrete groups. In (Ed. Dobrushin, R.L., Sinai, Ya.G.): Multicomponent random systems. Nauka, Moscow, 132-152 [English transl. (1980) Advances in probability and related topics, Vol. 6, 285-325. Marcel Dekker].

[5] Grigorchuk, R.I. (1983). On Milnor's problem of group growth. Dokl. Ak. Nauk SSSR, 271, 31-33 (Russian) [English transl. (1983): Soviet Math. Dokl., 28, 2326].

[6] Grigorchuk, R.I. (1990). On growth in group theory. Proc. of the International Congress of Mathematicians, Kyoto, 1990, 325-338.

[7] Gromov, M. (1981). Groups of polynomial growth and expanding maps. Publ. Math. IHES, 53, 53-73.

[8] Hall, M., Radó, T. (1948). On Schreier systems in free groups. Trans. AMS, 64, 386-408.

[9] Hall, M. (1949). Coset representations in free groups. Trans. AMS, 67, 421-432.

[10] Lewin, J. (1969). Free modules over free algebras and free group algebras : the Schreier technique. Trans. AMS, 145, 455-465.

[11] Lyndon, R.C., Schupp, P.E. (1977). Combinatorial group theory. Springer-Verlag.

[12] Milnor, J. (1968). A note on curvature and fundamental group. J. Diff. Geom., 2, $1-7$.

[13] Milnor, J. (1968). Growth of finitely generated solvable groups. J. Diff. Geom., 2, 447-449. 
[14] Rosenmann, A. Essentiality of fractal Ideals. Inter. J. Algebra and Computation (to appear).

[15] Rowen, L.H. (1988) Ring theory. Academic Press.

[16] Wolf, J.A. (1968). Growth of finitely generated solvable groups and curvature of Riemannian manifolds. J. Diff. Geom., 2, 421-446. 